\title{
Regulation of innate receptor pathways by microRNAs
}

\author{
ZHANG Yue ${ }^{1 *} \&$ LI YingKe ${ }^{2}$ \\ ${ }^{1}$ Department of General Surgery, East Hospital, Tongji University School of Medicine, Shanghai 200120, China; \\ ${ }^{2}$ Department of Anesthesiology, Changzheng Hospital, Second Military Medical University, Shanghai 200003, China
}

Received October 22, 2012; accepted November 26, 2012; published online December 20, 2012

\begin{abstract}
The innate immune response provides the initial defense against infection. This is accomplished by families of pattern recognition receptors (PRRs) that bind to conserved molecules in bacteria, fungi and viruses. PRRs are finely regulated by elaborate mechanisms to ensure a beneficial outcome in response to foreign invaders. MicroRNAs (miRNAs) are a class of small non-coding regulatory RNAs that are emerging as important regulators in immune responses at the post-transcriptional level, through the inhibition of translation, or by inducing mRNA degradation. It has been shown that miRNAs have unique expression profiles in cells of the innate immune systems and play pivotal roles in regulating the signal pathways of innate receptors, including Toll-like receptors, RIG-I-like receptors and Nod-like receptors. We have summarized the recent literature providing new insights into the regulation of innate receptor pathways by miRNAs.
\end{abstract}

innate receptor, microRNA, immune regulation

Citation: Zhang Y, Li Y K. Regulation of innate receptor pathways by microRNAs. Sci China Life Sci, 2013, 56: 13-18, doi: 10.1007/s11427-012-4428-2

Germline-encoded pattern-recognition receptors (PRRs) are microbial sensors of the host innate immune system. They recognize components of foreign pathogens, referred to as pathogen-associated molecular patterns (PAMPs). Several families of PRRs have been characterized, including Toll-like receptors (TLRs), RIG-I-like receptors (RLRs) and Nod-like receptors (NLRs) [1]. At least 13 different TLRs (10 in humans and 13 in mice) have been identified [2]. All TLRs have an extracellular sensing leucine-rich repeat (LRR) domain, a transmembrane domain, and a highly conserved cytoplasmic Toll- and interleukin-1-(IL-1) receptor (TIR). PAMPs recognized by TLRs include three general categories of ligands: proteins; nucleic acids; and lipid-based elements derived from a wide range of microbes such as bacteria, viruses, parasites and fungi. Two additional families of innate receptors, RLRs and NLRs, have been described and join the TLRs as key pathogen sensors [3]. The RLR family contains RIG-I, MDA5, and LGP2 as me-

*Corresponding author (email: magiczhangyue@yahoo.com.cn) mebers. These RLRs consist of two N-terminal caspase recruitment domains (CARDs), a central DEAD box helicase/ATPase domain, in addition to a C-terminal regulatory domain. RLRs recognize the double-stranded (ds) RNA generated as the replication intermediate of single-stranded (ss) RNA viruses, and the genomic RNA of dsRNA viruses [4]. NLRs are a group of intracellular microbial sensors that sense microbial products or the products of damaged cells, such as ATP and uric acid. NLRs are composed of a central nucleotide-binding domain and C-terminal leucine-rich repeats [5]. NLRs have been classified into four subfamilies with members such as NOD1, NOD2, NACHT, NALPs and IPAF. These PRRs have a critical role in both innate and adaptive immune responses [6].

Following the recognition of PAMPs, PRRs initiate innate immune responses by activating intracellular signaling cascades that lead to transcriptional expression of inflammatory mediators. These coordinate the elimination of pathogens and infected cells [7]. However, any dysregulation of this system leads to inflammatory diseases, autoim- 
mune diseases, or dissemination of pathogens. There is growing evidence to indicate that many molecules have been identified as positive or negative regulators of signaling for these innate receptors. For TLRs, these include membrane molecules (CD11b [8] and PECAM1 [9]), antigen presenting molecules (MHCI [10] and MHCII [11]), ubiquitin related proteins (Nrdp1 [12] and CHIP [13]), phosphatases (SHP-1 [14], SHP-2 [15], SHIP-1 [16] and PTP1B [17]), protein kinases (CaMKII [18]), endosome/lysosome-localized molecules (Rab7b [19,20], CLM [21]), a co-activator of gene transcription ( $\beta$-catenin [22]), HSP70 [23], HSP70L1 [24], and NGF [25]. The regulation of RLR and NLR signal pathways has been extensively investigated recently $[26,27]$; however, the precise mechanisms of regulation remain to be fully elucidated.

MicroRNAs (miRNAs) are noncoding RNAs with pleiotropic effects that are dependent on post-transcriptional and translational regulation of gene expression. These miRNA-related effects are essential for organ development, cell differentiation, and tumor progression [28,29]. MiRNAs are short ssRNAs of about 22 nucleotides, which regulate the expression of target genes at the post-transcriptional level by binding to their $3^{\prime}$ untranslated regions (UTRs). Around 1000 miRNAs have been identified in the human genome; more than $30 \%$ of protein-coding genes are predicted to be regulated by miRNAs at the post-transcriptional and translational level as each miRNA can potentially target thousands of different mRNAs. The biogenesis of miRNAs involves two processing steps by two RNaseIII enzymes, Drosha and Dicer. This then results in repression by translational inhibition, mRNA cleavage, and mRNA decay initiated by miRNA-guided deadenylation [30]. An accumulation of evidence has shown that miRNAs are involved in PRR signaling of the innate immune system. In this review, we aim to provide an overview of the regulation of innate receptor pathways by miRNAs, and how miRNAs can be used in the development of therapeutics against immune diseases.

\section{Regulation of TLR pathways by miRNAs}

Following ligand binding, TLRs initiate innate immune responses by activating downstream intracellular signaling pathways via the adaptor MyD88 or the adaptor TRIF. This induces the production of proinflammatory cytokines and type I interferon [31]. Many signal molecules, such as adaptor proteins and kinases, transcriptional factors, regulatory molecules, and inflammatory cytokines in the TLR signal pathway are identified to be likely targets of miRNAs, especially the TLR-induced miRNAs [32].

TLRs mainly signal through the MyD88-dependent pathway, which is responsible for the production of proinflammatory cytokines, and the TRIF-dependent pathway, leading to the anti-viral effects of TLRs. Previous reports have shown that tolerance is established and sustained by the activities of miR-146a, -155, -145, -346, -223 and -199a, which are known to target key adaptors and kinases of the TLR signaling pathway. The miR-146 family is composed of two members, miR-146a and miR-146b. MiR-146a is one of the most prominent miRNAs induced by TLR signals through NF-KB activation, and then feeds back to suppress TLR-triggered NF- $\mathrm{KB}$ activation [33]. Like miR-146a, miR-155 is known to target the TAK1-binding protein 2 (TAB2), inhibiting the activation of TAK1, and hence NF- $\mathrm{KB}$ and MAPK, thereby acting as an anti-inflammatory agent [34]. Significant targets of miR-155 include IKKe, FADD and RIP-1 [35]. The MyD88 adaptor-like protein (MAL), which functions as a bridging adaptor for TLR2and TLR4-mediated MyD88-dependent signaling and undergoes proteasomal degradation following TLR2 and TLR4 stimulation, has emerged as a target of miR-145 [36]. Bruton's tyrosine kinase (BTK) participates in the TLR4, TLR7-TLR8 and TLR9 signaling pathways required for NF- $\mathrm{KB}$ activation, and it has recently been shown that miR-346 is strongly induced by treatment with lipopolysaccharides (LPS). miR-346 has been shown to target Btk mRNA in synovial fibroblasts (FLS) of rheumatoid arthritis (RA) patients. Whether miR-346 in macrophages targets Btk mRNA needs to be further studied [37]. In macrophages, a decrease in miR-15a, -16, and -223 expression levels has been shown to correlate with an increase in IKK $\alpha$ protein expression, suggesting that these miRNAs could function as modulators of IKK $\alpha$ mRNA and protein expression [38]. Chen $\mathrm{R}$ et al. identified miR-199a as a regulator of IKK $\beta$ which is a major factor promoting a functional TLR-MyD88NF- $\mathrm{KB}$ pathway to constitutively secrete proinflammatory/protumor cytokines [39]. A link between dysregulation of miRNAs and human brain disorders has become increasingly evident [40,41]. Recently, extracellular let-7 has been shown to activate the RNA-sensing TLR7 and induce neurodegeneration [42].

The stimulation of TLRs delivers signals through adaptor molecules and kinases. Ultimately, activation of certain transcription factors in the nucleus is the key step to triggering target gene transcription. Targeting of transcription factors by miRNAs possibly has an impact on TLR-induced gene expression [43]. The most important transcription factor of the TLR signaling pathway, NF- $\mathrm{kB}$, is present in the cytoplasm, in an inactive form, and is trapped by an inhibi-

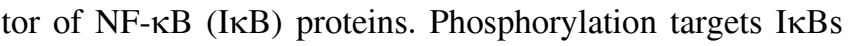
for ubiquitination and degradation, allowing NF- $\mathrm{KB}$ to be released into the nucleus and to bind to a response element, which starts transcription of the target genes. MiR-9, the TLR-responsive miRNA in the MyD88- and NF- $\mathrm{KB}-$ dependent pathways, is shown to directly target Nfkb1 mRNA and then control the NF- $\mathrm{KB}$ responses by feedback [44]. More recently, miR-210 has been shown to be induced by LPS and target Nfkb1 mRNA [45]. The signal transduc- 
tion and activator of transcription (STAT)-mediated feedback loop amplifies signaling by cytokines, and enhances macrophage responses to microbial inducers like TLR ligands [46]. The expression of STAT3 is found to be modulated by both miR-17-5p and miR-20a, suggesting that miRNAs could potentially be used for immunotherapy against diseases by blocking STAT3 expression [47]. Peroxisome proliferator-activated receptor $\gamma$ (PPAR $\gamma$ ) belongs to the nuclear hormone receptor superfamily of ligand-activated transcription factors and originally has been characterized to be an important therapeutic target of chronic inflammatory diseases. Jennewein et al. recently provided evidence that LPS-induced miR-27b contributes to destabilization of PPAR $\gamma 1$ mRNA, suggesting that decreased PPAR $\gamma$ expression by miRNAs prolongs inflammation, thereby attenuating resolution of inflammation [48]. Moreover, the transcriptional co-repressor $\mathrm{C} / \mathrm{EBP} \beta$ is shown to be targeted by miR-155, and the transcriptional co-activator $\mathrm{p} 300$ is targeted by miR-132 $[49,50]$.

In addition to directly regulating adaptor molecules and kinases, as well as transcription factors of the TLR signaling pathway, it is increasingly apparent that miRNAs can target the regulators of TLR signaling. MiR-21 is expressed in numerous cancers and has been well established as an 'oncomiR'. Furthermore, miR-21 is implicated as a central player in the inflammatory response. The tumor suppressor protein programmed cell death 4 (PDCD4), an inhibitor of translation by targeting eukaryotic translation-initiation factor $4 \mathrm{~F}$ (EIF4F), which is required for the initiation of translation at the 5' UTR of mRNA sequences, has been recently identified to be a key target of miR-21 during TLR4 signaling in macrophages [51]. Iliopoulos et al. found that miR-21 targets the PTEN tumor suppressor gene that functions through the Akt pathway, further underlining the significance of miR-21 in inflammation and cancer [52]. Notch1 has also been identified as a target of miR-146a in studies of the miR-146a-mediated repression of IL-12p70 production in TLR9-triggered DCs [53]. O'Connell et al. showed that Src homology-2 domain-containing inositol 5-phosphatase 1 (SHIP1) is a direct target of miR-155, and the repression of endogenous SHIP1 by miR-155 results in increased activation of the kinase Akt during the cellular response to LPS [54].

Activation of TLRs leads to the induction of inflammatory cytokines such as TNF, IL-6, IL-12, and IL-10, and chemokines such as MCP-1, MIP-1 $\alpha$, IP-10 and RANTES $[55,56]$, thereby linking the innate and adaptive immune systems. Several miRNAs, such as miR-221, miR-125b and miR-939 have been found to target the 3' UTR of TNF- $\alpha$ mRNA during LPS tolerance [57,58]. Recently, miR-365, miR-142-3p and let-7 were found to target I16 mRNA, and reduced endotoxin-induced mortality. Lu et al. identified mIL-12p35 as a molecular target of miR-21, which probably contributes to polarization of $T$ helper $\left(T_{h}\right)$ cells, sug- gesting a key role for miRNAs in allergic lung inflammation [59]. More recently, miR-466l has been shown to up-regulate IL-10 expression in TLR-triggered macrophages [60]. Ma et al. found that miR-29 suppresses immune responses to intracellular pathogens by directly targeting IFN- $\gamma[61]$.

\section{Regulation of RLR pathways by miRNAs}

The RLR sensors recognize the genetic material of RNA viruses in the cytoplasm of infected cells and induce inflammatory cytokines and type I interferons. Upon recognition, conformational changes within these sensors exposing the CARD domains of RIG-I and MDA5 are induced. They then then interact with the CARD-containing adaptor protein, IPS-1. IPS-1 localizes to the mitochondria and is required for downstream signaling to activate IKK-related kinases, TBK1/IKKi, which then activates IRF3/IRF7 and the subsequent transcription of type I interferons via TRAF3. Upon recruitment, IPS-1 also activates NF- $\kappa \mathrm{B}$ through recruitment of TRADD, FADD, caspase- 8 , and caspase-10 [4].

Several miRNAs can be induced by RIG-I signaling, and regulate viral replication through modulating the RIG-I pathway and the expression of type I interferon. miR-146a has been shown to regulate vesicular stomatitis virus (VSV) infection-triggered type I IFN production by negatively feeding back and targeting TRAF6, IRAK1 and IRAK2 [62]. The stimulator of IFN genes (STING) protein is a recently discovered adaptor protein that functions downstream of RIG-I and upstream of TBK1 and plays an important role in IFN- $\beta$ production. Huang et al. found that endogenous STING could be regulated post-transcriptionally by miR-24 in IEC-6 cells [63]. Interferon- $\alpha$ (IFN- $\alpha$ ) is a critical molecular mediator of pathogen-induced immune responses [64]. Recently, miR-4661 has been demonstrated to directly target IFN- $\alpha$ expression through the $3^{\prime}$ UTR, and inhibit host antiviral innate immune responses in Sendai virus $(\mathrm{SeV})$ - and VSV-infected macrophages and dendritic cells [65]. Li et al. recently found that miR-122 facilitates replication of hepatitis $\mathrm{C}$ virus (HCV) RNA and may partly counteract the anti-HCV effect of IFN- $\alpha$ [66]. MiR-29a has recently been identified to be critical for diminishing the sensitivity of the thymic epithelium in stimulating infection signals, protecting the thymus against inappropriate involution via miR-29a-mediated suppression of the IFN- $\alpha$ receptor [67]. Furthermore, using an in-depth analysis of miRNomes in resting and IFN- $\alpha$-activated human natural killer (NK) cells, two abundant miRNAs (miR-378 and miR-30e) have been shown to be suppressed by IFN- $\alpha$ activation. They then directly target granzyme B and perforin [68].

Some viral miRNAs can regulate viral and/or host cell 
gene expression. MiR-H2-3p, expressed by herpes simplex virus 1 (HSV-1), is now known to affect ICP0, a viral immediate-early transcriptional activator thought to play a key role in productive $\mathrm{HSV}-1$ replication and reactivation from latency [69]. Liang et al. have recently found that miR-K12-11, encoded by Kaposi's sarcoma-associated herpesvirus (KSHV) is critical for the modulation of IFN signaling and contributes to maintenance of KSHV latency by targeting IKKe [70].

\section{Regulation of NLR pathways by miRNAs}

Ligation of NOD1 or NOD2 with related ligands activates MAPK- and NF- $\kappa \mathrm{B}$-signaling cascades, leading to the production of proinflammatory cytokines and chemokines. Other NLRs are involved in the assembly and activation of inflammasomes, which control the activation of caspase-1, and subsequent processing of IL-1 $\beta$ and IL-18 [71]. Haneklaus et al. identified that a highly conserved miR-223 targets the site of the NLRP3 3' UTR and prevents accumulation of NLRP3 protein, and inhibits IL-1 $\beta$ production from the inflammasome. Furthermore, Epstein-Barr virus (EBV) can also take advantage of miR-BART15 to limit inflammation [72]. Bauernfeind et al. also found that miR-223 functions to control NLRP3 inflammasome activity [73]. It is possible there may be other miRNAs involved in the regulation of NLR pathways, but this requires further investigation.

\section{Conclusion and perspective}

The goal of inflammation is to resolve an injury and return the immune system to homeostasis. Many studies have focused on cellular and molecular networks that regulate inflammation, and recent breakthroughs have been made in understanding the roles of miRNAs in innate immunity. These miRNAs regulate innate receptor signaling pathways and ensuing cytokine responses at multiple epigenetic levels. Although the study of miRNAs has opened up many new areas in the regulation of innate receptor signaling, the mechanistic insight into the precise effects of miRNAs on TLR-, RLR- and NLR-mediated inflammatory responses are still lacking. In particular, how these miRNA networks collaborate together to optimize immune responses requires further elucidation. Future studies will illuminate the link between changes in miRNA levels and the onset of human inflammatory diseases. It is hoped this will lead to using miRNAs in diagnostics and therapeutics for the treatment of inflammatory diseases, autoimmunity, and cancers [74,75].

1 Takeuchi O, Akira S. Pattern recognition receptors and inflammation. Cell, 2010, 140: 805-820

2 Netea M G, Wijmenga C, O'Neill L A. Genetic variation in Toll-like receptors and disease susceptibility. Nat Immunol, 2012, 13: 535-542

3 Kawai T, Akira S. The roles of TLRs, RLRs and NLRs in pathogen recognition. Int Immunol, 2009, 21: 317-337

4 Loo Y M, Gale M Jr. Immune signaling by RIG-I-like receptors. Immunity, 2011, 34: 680-692

5 Maekawa T, Kufer T A, Schulze-Lefert P. NLR functions in plant and animal immune systems: so far and yet so close. Nat Immunol, 2011, 12: 817-826

6 Hancock R E, Nijnik A, Philpott D J. Modulating immunity as a therapy for bacterial infections. Nat Rev Microbiol, 2012, 10: 243-254

7 Qian C, Cao X. Regulation of Toll-like receptor signaling pathways in innate immune responses. Ann N Y Acad Sci, 2012, doi: 10.1111/j1749-6632.2012.06786.x

8 Han $\mathrm{C}$, Jin $\mathrm{J}, \mathrm{Xu} \mathrm{S}$, et al. Integrin $\mathrm{CD} 11 \mathrm{~b}$ negatively regulates TLR-triggered inflammatory responses by activating Syk and promoting degradation of MyD88 and TRIF via Cbl-b. Nat Immunol, 2010, 11: 734-742

9 Rui Y, Liu X, Li N, et al. PECAM-1 ligation negatively regulates TLR4 signaling in macrophages. J Immunol, 2007, 179: 7344-7351

10 Xu S, Liu X, Bao Y, et al. Constitutive MHC class I molecules negatively regulate TLR-triggered inflammatory responses via the Fps-SHP-2 pathway. Nat Immunol, 2012, 13: 551-559

11 Liu X, Zhan Z, Li D, et al. Intracellular MHC class II molecules promote TLR-triggered innate immune responses by maintaining activation of the kinase Btk. Nat Immunol, 2011, 12: 416-424

12 Wang $\mathrm{C}$, Chen T, Zhang J, et al. The E3 ubiquitin ligase Nrdp1 'preferentially' promotes TLR-mediated production of type I interferon. Nat Immunol, 2009, 10: 744-752

13 Yang M, Wang C, Zhu X, et al. E3 ubiquitin ligase CHIP facilitates Toll-like receptor signaling by recruiting and polyubiquitinating $\mathrm{Src}$ and atypical PKC\{zeta\}. J Exp Med, 2011, 208: 2099-2112

14 An H, Hou J, Zhou J, et al. Phosphatase SHP-1 promotes TLR- and RIG-I-activated production of type I interferon by inhibiting the kinase IRAK1. Nat Immunol, 2008, 9: 542-550

15 An H, Zhao W, Hou J, et al. SHP-2 phosphatase negatively regulates the TRIF adaptor protein-dependent type I interferon and proinflammatory cytokine production. Immunity, 2006, 25: 919-928

16 An $\mathrm{H}, \mathrm{Xu} \mathrm{H}$, Zhang M, et al. Src homology 2 domain-containing inositol-5-phosphatase 1 (SHIP1) negatively regulates TLR4-mediated LPS response primarily through a phosphatase activity- and PI-3K-independent mechanism. Blood, 2005, 105: 4685-4692

$17 \mathrm{Xu} \mathrm{H}$, An H, Hou J, et al. Phosphatase PTP1B negatively regulates MyD88- and TRIF-dependent proinflammatory cytokine and type I interferon production in TLR-triggered macrophages. Mol Immunol, 2008, 45: 3545-3552

18 Liu X, Yao M, Li N, et al. CaMKII promotes TLR-triggered proinflammatory cytokine and type I interferon production by directly binding and activating TAK1 and IRF3 in macrophages. Blood, 2008, 112: 4961-4970

19 Yao M, Liu X, Li D, et al. Late endosome/lysosome-localized Rab7b suppresses TLR9-initiated proinflammatory cytokine and type I IFN production in macrophages. J Immunol, 2009, 183: 1751-1758

20 Wang Y, Chen T, Han C, et al. Lysosome-associated small Rab GTPase Rab7b negatively regulates TLR4 signaling in macrophages by promoting lysosomal degradation of TLR4. Blood, 2007, 110: 962-971

21 Wu Y, Zhu X, Li N, et al. CMRF-35-like molecule 3 preferentially promotes TLR9-triggered proinflammatory cytokine production in macrophages by enhancing TNF receptor-associated factor 6 ubiquitination. J Immunol, 2011, 187: 4881-4889

22 Yang P, An H, Liu X, et al. The cytosolic nucleic acid sensor LRRFIP1 mediates the production of type I interferon via a betacatenin-dependent pathway. Nat Immunol, 2010, 11: 487-494

23 Chen T, Guo J, Han C, et al. Heat shock protein 70, released from heat-stressed tumor cells, initiates antitumor immunity by inducing tumor cell chemokine production and activating dendritic cells via TLR4 pathway. J Immunol, 2009, 182: 1449-1459

24 Fang H, Wu Y, Huang X, et al. Toll-like receptor 4 (TLR4) is essen- 
tial for Hsp70-like protein 1 (HSP70L1) to activate dendritic cells and induce Th1 response. J Biol Chem, 2011, 286: 30393-30400

25 Jiang Y, Chen G, Zhang Y, et al. Nerve growth factor promotes TLR4 signaling-induced maturation of human dendritic cells in vitro through inducible p75NTR 1. J Immunol, 2007, 179: 6297-6304

26 Ramos H J, Gale M Jr. RIG-I like receptors and their signaling crosstalk in the regulation of antiviral immunity. Curr Opin Virol, 2011, 1: 167-176

27 Bonardi V, Cherkis K, Nishimura M T, et al. A new eye on NLR proteins: focused on clarity or diffused by complexity? Curr Opin Immunol, 2012, 24: 41-50

28 Ebert M S, Sharp P A. Roles for microRNAs in conferring robustness to biological processes. Cell, 2012, 149: 515-524

29 Yang C, Wei W. The miRNA expression profile of the uveal melanoma. Sci China Life Sci, 2011, 54: 351-358

30 Mendell J T, Olson E N. MicroRNAs in stress signaling and human disease. Cell, 2012, 148: 1172-1187

31 Kawai T, Akira S. The role of pattern-recognition receptors in innate immunity: update on Toll-like receptors. Nat Immunol, 2010, 11: 373-384

32 O'Neill L A, Sheedy F J, McCoy C E. MicroRNAs: the fine-tuners of Toll-like receptor signalling. Nat Rev Immunol, 2011, 11: 163-175

33 Taganov K D, Boldin M P, Chang K J, et al. NF-kappaB-dependent induction of microRNA miR-146, an inhibitor targeted to signaling proteins of innate immune responses. Proc Natl Acad Sci USA, 2006, 103: $12481-12486$

34 Ceppi M, Pereira P M, Dunand-Sauthier I, et al. MicroRNA-155 modulates the interleukin-1 signaling pathway in activated human monocyte-derived dendritic cells. Proc Natl Acad Sci USA, 2009, 106: 2735-2740

35 Tili E, Michaille J J, Cimino A, et al. Modulation of miR-155 and miR-125b levels following lipopolysaccharide/TNF-alpha stimulation and their possible roles in regulating the response to endotoxin shock. J Immunol, 2007, 179: 5082-5089

36 Starczynowski D T, Kuchenbauer F, Argiropoulos B, et al. Identification of miR-145 and miR-146a as mediators of the 5q-syndrome phenotype. Nat Med, 2010, 16: 49-58

37 Alsaleh G, Suffert G, Semaan N, et al. Bruton's tyrosine kinase is involved in miR-346-related regulation of IL-18 release by lipopolysaccharide-activated rheumatoid fibroblast-like synoviocytes. J Immunol, 2009, 182: 5088-5097

38 Li T, Morgan M J, Choksi S, et al. MicroRNAs modulate the noncanonical transcription factor NF-kappaB pathway by regulating expression of the kinase IKKalpha during macrophage differentiation. Nat Immunol, 2010, 11: 799-805

39 Chen R, Alvero A B, Silasi D A, et al. Regulation of IKKbeta by miR-199a affects NF-kappaB activity in ovarian cancer cells. Oncogene, 2008, 27: 4712-4723

40 Feng W, Feng Y. MicroRNAs in neural cell development and brain diseases. Sci China Life Sci, 2011, 54: 1103-1112

41 Li S, Yu B, Wang Y, et al. Identification and functional annotation of novel microRNAs in the proximal sciatic nerve after sciatic nerve transection. Sci China Life Sci, 2011, 54: 806-812

42 Lehmann S M, Krüger C, Park B, et al. An unconventional role for miRNA: let-7 activates Toll-like receptor 7 and causes neurodegeneration. Nat Neurosci, 2012, 15: 827-835

43 Chen K, Rajewsky N. The evolution of gene regulation by transcription factors and microRNAs. Nat Rev Genet, 2007, 8: 93-103

44 Bazzoni F, Rossato M, Fabbri M, et al. Induction and regulatory function of miR-9 in human monocytes and neutrophils exposed to proinflammatory signals. Proc Natl Acad Sci USA, 2009, 106: 5282-5287

45 Qi J, Qiao Y, Wang P, et al. microRNA-210 negatively regulates LPS-induced production of proinflammatory cytokines by targeting NF-kappaB1 in murine macrophages. FEBS Lett, 2012, 586: 1201-1207

46 Hu X, Chen J, Wang L, et al. Crosstalk among Jak-STAT, Toll-like receptor, and ITAM-dependent pathways in macrophage activation. J Leukoc Biol, 2007, 82: 237-243
47 Zhang M, Liu Q, Mi S, et al. Both miR-17-5p and miR-20a alleviate suppressive potential of myeloid-derived suppressor cells by modulating STAT3 expression. J Immunol, 2011, 186: 4716-4724

48 Jennewein C, von Knethen A, Schmid T, et al. MicroRNA-27b contributes to lipopolysaccharide-mediated peroxisome proliferatoractivated receptor gamma (PPARgamma) mRNA destabilization. J Biol Chem, 2010, 285: 11846-11853

49 Lagos D, Pollara G, Henderson S, et al. miR-132 regulates antiviral innate immunity through suppression of the p300 transcriptional co-activator. Nat Cell Biol, 2010, 12: 513-519

50 Worm J, Stenvang J, Petri A, et al. Silencing of microRNA-155 in mice during acute inflammatory response leads to derepression of c/ebp Beta and down-regulation of G-CSF. Nucleic Acids Res, 2009, 37: 5784-5792

51 Sheedy F J, Palsson-McDermott E, Hennessy E J, et al. Negative regulation of TLR4 via targeting of the proinflammatory tumor suppressor PDCD4 by the microRNA miR-21. Nat Immunol, 2010, 11: $141-147$

52 Iliopoulos D, Jaeger S A, Hirsch H A, et al. STAT3 activation of miR-21 and miR-181b-1 via PTEN and CYLD are part of the epigenetic switch linking inflammation to cancer. Mol Cell, 2010, 39: 493-506

53 Bai Y, Qian C, Qian L, et al. Integrin CD11b negatively regulates TLR9-triggered dendritic cell cross-priming by upregulating microRNA-146a. J Immunol, 2012, 188: 5293-5302

54 O'Connell R M, Chaudhuri A A, Rao D S, et al. Inositol phosphatase SHIP1 is a primary target of miR-155. Proc Natl Acad Sci USA, 2009, 106: 7113-7118

55 Qian C, Jiang X, An H, et al. TLR agonists promote ERK-mediated preferential IL-10 production of regulatory dendritic cells (diffDCs), leading to NK-cell activation. Blood, 2006, 108: 2307-2315

56 Qian C, An H, Yu Y, et al. TLR agonists induce regulatory dendritic cells to recruit Th1 cells via preferential IP-10 secretion and inhibit Th1 proliferation. Blood, 2007, 109: 3308-3315

57 Semaan N, Frenzel L, Alsaleh G, et al. miR-346 controls release of TNF-alpha protein and stability of its mRNA in rheumatoid arthritis via tristetraprolin stabilization. PLoS One, 2011, 6: 1-11

58 El G M, McCall C E. MicroRNAs distinguish translational from transcriptional silencing during endotoxin tolerance. J Biol Chem, 2010, 285: 20940-20951

59 Lu T X, Munitz A, Rothenberg M E. MicroRNA-21 is up-regulated in allergic airway inflammation and regulates IL-12p35 expression. J Immunol, 2009, 182: 4994-5002

60 Ma F, Liu X, Li D, et al. MicroRNA-4661 upregulates IL-10 expression in TLR-triggered macrophages by antagonizing RNA-binding protein tristetraprolin-mediated IL-10 mRNA degradation. J Immunol, 2010, 184: 6053-6059

61 Ma F, Xu S, Liu X, et al. The microRNA miR-29 controls innate and adaptive immune responses to intracellular bacterial infection by targeting interferon-gamma. Nat Immunol, 2011, 12: 861-869

62 Hou J, Wang P, Lin L, et al. MicroRNA-146a feedback inhibits RIG-I-dependent Type I IFN production in macrophages by targeting TRAF6, IRAK1, and IRAK2. J Immunol, 2009, 183: 2150-2158

63 Huang Z, Chen X, Yu B, et al. Cloning and functional characterization of rat stimulator of interferon genes (STING) regulated by miR-24. Dev Comp Immunol, 2012, 37: 414-420

64 MacMicking J D. Interferon-inducible effector mechanisms in cell-autonomous immunity. Nat Rev Immunol, 2012, 12: 367-382

65 Li Y, Fan X, He X, et al. MicroRNA-4661 inhibits antiviral innate immune response by targeting interferon-alpha. Cell Mol Immunol, 2012, doi: $10.1038 / \mathrm{cmi} .2012 .35$

66 Li S J, Chen Z, Zhu H H. Influence of miR-122 on IFN-alpha treatment for HCV infection. Zhejiang Da Xue Xue Bao Yi Xue Ban, 2011, 40: 588-592

67 Papadopoulou A S, Dooley J, Linterman M A, et al. The thymic epithelial microRNA network elevates the threshold for infection-associated thymic involution via miR-29a mediated suppression of the IFN-alpha receptor. Nat Immunol, 2012, 13: 181-187

68 Wang P, Gu Y, Zhang Q, et al. Identification of resting and type I IFN-activated human NK cell miRNomes reveals microRNA-378 
and microRNA-30e as negative regulators of NK cell cytotoxicity. $\mathrm{J}$ Immunol, 2012, 189: 211-221

69 Umbach J L, Kramer M F, Jurak I, et al. MicroRNAs expressed by herpes simplex virus 1 during latent infection regulate viral mRNAs. Nature, 2008, 454: 780-783

70 Liang D, Gao Y, Lin X, et al. A human herpesvirus miRNA attenuates interferon signaling and contributes to maintenance of viral latency by targeting IKKe. Cell Res, 2011, 21: 793-806

71 Franchi L, Munoz-Planillo R, Nunez G. Sensing and reacting to microbes through the inflammasomes. Nat Immunol, 2012, 13: 325-332
72 Haneklaus M, Gerlic M, Kurowska-Stolarska M, et al. Cutting edge: miR-223 and EBV miR-BART15 regulate the NLRP3 inflammasome and IL-1beta production. J Immunol, 2012, 189: 3795-3799

73 Bauernfeind F, Rieger A, Schildberg F A, et al. NLRP3 inflammasome activity is negatively controlled by miR-223. J Immunol, 2012, 189: 4175-4181

74 O'Connell R M, Rao D S, Baltimore D. microRNA regulation of inflammatory responses. Annu Rev Immunol, 2012, 30: 295-312

75 Feng Y, Yu X. Cardinal roles of miRNA in cardiac development and disease. Sci China Life Sci, 2011, 54: 1113-1120

Open Access This article is distributed under the terms of the Creative Commons Attribution License which permits any use, distribution, and reproduction in any medium, provided the original author(s) and source are credited. 